\title{
Review of theoretical methods and research aspects for detecting leaf water content using terahertz spectroscopy and imaging
}

\author{
Fangfang $\mathrm{Qu}^{1,2}$, Pengcheng $\mathrm{Ni}^{1,2,3}$, Lei $\mathrm{Lin}^{1,2}$, Chengyong Cai ${ }^{1,2}$, Yong $\mathrm{He}^{1,2^{*}}$ \\ (1. College of Biosystems Engineering and Food Science, Zhejiang University, Hangzhou 310058, China; \\ 2. Key Laboratory of Spectroscopy Sensing, Ministry of Agriculture and Rural Affairs, Hangzhou 310058, China; \\ 3. State Key Laboratory of Modern Optical Instrumentation, College of Optical Science and Engineering, \\ Zhejiang University, Hangzhou 310058, China)
}

\begin{abstract}
The water content in vegetative leaves is an important indicator to plant science. It reveals the physiological status of plants and provides valuable information in irrigation management. Terahertz (THz) as a state-of-the-art technology shows great potential in measuring and monitoring the water status in plant leaves. This paper reviewed the theoretical models for calculating water content in the plant leaves, the methods for eliminating the scattering loss caused by the surface roughness of leaf, the applications of $\mathrm{THz}$ spectroscopy and $\mathrm{THz}$ imaging for monitoring leaf water content and describing leaf water distribution. The survey of the researches presents the considerable advantages of this emerging and promising $\mathrm{THz}$ technology in agriculture.
\end{abstract}

Keywords: terahertz (THz) spectroscopy, terahertz imaging, leaf water content, leaf water distribution, theoretical models, eliminating scattering loss, agriculture

DOI: $10.25165 /$ j.ijabe.20181105.3952

Citation: Qu F F, Nie P C, Lin L, Cai C Y, He Y. Review of theoretical methods and research aspects for detecting leaf water content using terahertz spectroscopy and imaging. Int J Agric \& Biol Eng, 2018; 11(5): 27-34.

\section{Introduction}

Water is an essential component in the physiological process of plants, which participates in the various activities such as hydrolysis, photosynthesis, transpiration, and nutrient transport ${ }^{[1,2]}$. The leaf water content is an important indicator to measure plant status. Proper amount of water is required for plant growth. Otherwise, the normal physiological processes of the plant will be disturbed $^{[3]}$. It will cause a series of reactions such as inhibition of aerobic respiration and reduction of water potential and turgor pressure $^{[4]}$. It will even cause some plant diseases, such as leaf chlorosis, necrosis and wilt ${ }^{[5,6]}$. Furthermore, air pollution and pesticide spraying may change the content and distribution of water in plant leaves ${ }^{[7,8]}$. Therefore, the detection of leaf water content and distribution is of high importance for numerous aspects in plant science including basic research and plant biology ${ }^{[9]}$. It provides valuable information in irrigation management and physiological condition and helps to avoid plant drought stress ${ }^{[10,11]}$.

State-of-the-art techniques in determining leaf water status can be divided into destructive detection and nondestructive detection. The destructive detection methods include thermogravimetric, distillation, Carle Fischer, psychrometers, pressure chambers, gas exchange systems, etc. ${ }^{[12-14]}$ These detection methods are

\section{Received date: 2017-11-03 Accepted date: 2018-08-16}

Biographies: Fangfang $\mathrm{Qu}, \mathrm{PhD}$ candidate, research interest: precision agriculture and terahertz technology, Email: ffqu@zju.edu.cn; Pengcheng Nie, Associate Research Fellow, research interest: internet of things technology and intelligent agricultural equipment, Email: npc2012@zju.edu.cn; Lei Lin, PhD candidate, research interest: Raman and terahertz technology, Email: linlei2016@zju.edu.cn; Chengyong Cai, Master Candidate, research interest: terahertz technology, Email: alayy@zju.edu.cn.

*Corresponding author: Yong He, Professor, research interests: intelligent agricultural technology, precision agriculture. College of Biosystems Engineering and Food Science, Zhejiang University, 866 Yuhangtang Rd, Hangzhou 310058, China. Tel/Fax: +86-571-88982143, Email: yhe@zju.edu.cn. generally time-consuming and energy-costing in preparation of the samples. The validity of the data and the synchronization between different measurements cannot be guaranteed. For long-term studies of the plant, nondestructive and contactless methods are required. One of the most common techniques used for nondestructive detection is based on spectroscopy. With the development of spectral technology, visible, near, mid, short-wave, thermal infrared, and hyperspectral images are applied as adequate analytical tools for leaf water detection ${ }^{[15-17]}$. However, these methods usually require to select the spectral variables that sensitive to water. Nuclear magnetic resonance (NMR) has also been adopted for determination of leaf water content. But this approach requires complex systems and is not suitable for the development of compact instruments ${ }^{[18]}$. Terahertz (THz) technology is an emerging, non-destructive and real-time detection technology. Because the vibrational and rotational energy levels of many molecules and the weak intermolecular interactions are in the terahertz band, it has a unique advantage in detection of water according to the fingerprint characteristics. Besides, phase information and amplitude information can be obtained during the detection. The strong attenuation of terahertz radiation by water makes $\mathrm{THz}$ a very sensitive non-contact probe of performing nondestructive in-vivo detection of the leaf water content ${ }^{[19,20]}$. Additionally, the $\mathrm{THz}$ technique has the advantage of excellent signal-to-noise performance. Due to its superiority, the $\mathrm{THz}$ technique has been used as an effective tool in the field of agriculture.

\section{Terahertz radiation}

The THz frequencies are in the range of $0.1-10 \mathrm{THz}(0.03-$ $3 \mathrm{~mm})$. The main parameters related to THz are listed in Table 1. In the electromagnetic spectrum, $\mathrm{THz}$ is in the transition region between infrared and millimeter wave, which makes $\mathrm{THz}$ has the electronic and optical properties simultaneously ${ }^{[21,2]}$. 
Table 1 Main parameters related to $\mathbf{T H z}$

\begin{tabular}{cl}
\hline Item & \multicolumn{1}{c}{ Equation } \\
\hline Frequency & $v=1 \mathrm{THz}=1000 \mathrm{GHz}=10^{12} \mathrm{~Hz}$ \\
Angular period & $\omega=2 \pi v=6.28 \mathrm{THz}$ \\
Cycle period & $\tau=1 / v=1 \mathrm{ps}=10^{-12} \mathrm{~s}$ \\
Wavelength & $\lambda=c / v=0.3 \mathrm{~mm}=300 \mathrm{um}$ \\
Wavenumber & $k=1 / \lambda=33.3 \mathrm{~cm}^{-1}$ \\
Photon energy & $h v=4.14 \mathrm{meV}^{-10}$ \\
Temperature & $T=h v / k=48 \mathrm{~K}$ \\
\hline
\end{tabular}

An abundant of physical and chemical information of the tested materials is contained in the terahertz spectra. Because of the intermolecular interactions including hydrogen bonding, van der Walls force and dipole rovibrational transitions, crystal lattice vibration can be reflected in the terahertz radiation ${ }^{[23]}$. Most polar molecules and many organic molecules exhibit strong absorption and dispersion properties in the terahertz band, which is beneficial to the study of the molecular structure by analyzing their terahertz spectra $^{[24-26]}$. The solid biomaterials have their own characteristic absorption in terahertz band (the effective spectral range of the present report is generally $0-3 \mathrm{THz}$ ), and its characteristic absorption mainly comes from the collective vibration mode of the molecule ${ }^{[27,28]}$. Some quantum chemical methods can be applied to calculate the vibration absorption spectra of molecules in terahertz band, including density functional theory (DFT) ${ }^{[29]}$, ab initio theory $(\mathrm{HF})^{[30,31]}$, semi empirical algorithm ${ }^{[32]}$. Compared to other spectrum such as microwave, visible/ near/ mid/ thermal infrared, there are several unique properties of $\mathrm{THz}$, such as transmittance property, low energy property, water absorption property, transient property, coherent property, and fingerprint spectrum. As a result, terahertz radiation has widespread potential to be applied in scientific researches and in the application fields of chemistry, physics, biology and agriculture ${ }^{[33]}$.

\section{Terahertz spectroscopy and imaging systems}

The technologies of terahertz spectroscopy and terahertz imaging provide an efficient method to detect the water status in plant leaves ${ }^{[34]}$. Since water is a broadband absorber without notable spectral features at terahertz and sub-terahertz frequencies, the water content and response of the water stress of the tested leaf can be detected by simply evaluating the peak-to-peak amplitude of the time domain spectroscopy. And the water distribution can be depicted by a continuous wave terahertz imaging at any frequency.

\subsection{Terahertz spectroscopy system}

Terahertz time-domain spectroscopy (THz-TDS) is an emerging and effective coherent detection technology. It is a complementary to infrared and Raman spectroscopy ${ }^{[35]}$. Compared with time-resolved terahertz spectroscopy and terahertz emission spectroscopy, THz-TDS is the most typical $\mathrm{THz}$ spectroscopy and received wide attention and application. The detection modes of $\mathrm{THz}$ Time-Domain Spectrometer include transmission, reflection, differential, ellipsometry, etc. Among these modes, the transmission and reflection THz-TDS system are most commonly used. At present, there are two main methods of terahertz pulse generation: photoconductive antenna and optical rectification. The terahertz pulse detection methods are photoconductive sampling and electro-optic sampling ${ }^{[36]}$. The typical THz-TDS system is shown in Figure 1. It is consisted of femtosecond laser, terahertz radiation generator, detector and time delay control system. The ultrafast laser is split into a pump beam and a probe beam by the beam splitter. The pump beam is incident on the terahertz emission crystal through a variable delay line to generate terahertz pulses. The terahertz pulses are focused on the detection crystal by two sets of off-axis parabolic mirrors. The probe beam is used to gate the detector and measure the instantaneous terahertz electric field. A delay stage is used to offset the pump and probe beams and allow the terahertz temporal profile to be iteratively sampled.

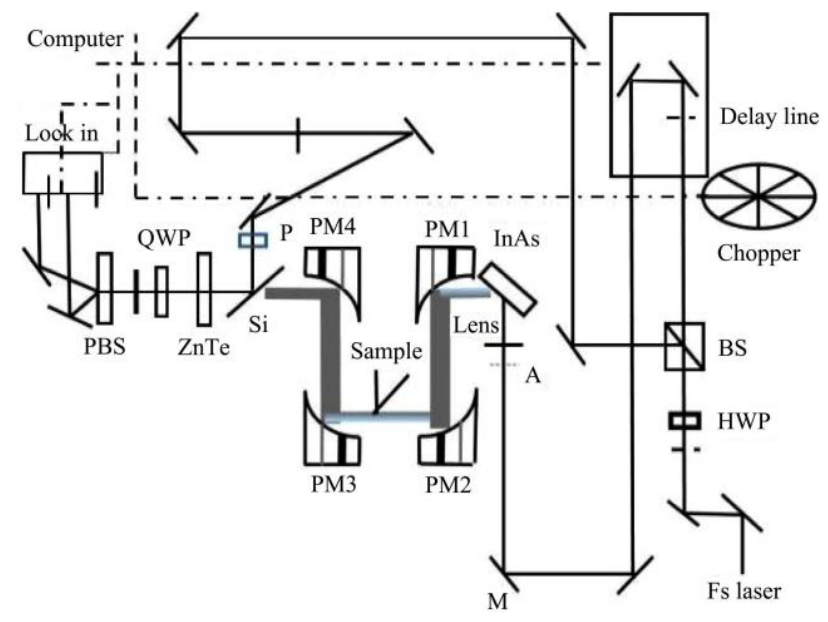

a. Schematic diagram of THz-TDS system

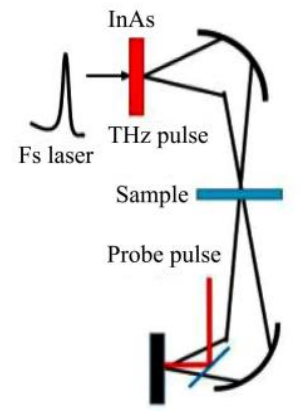

b. Transmission mode

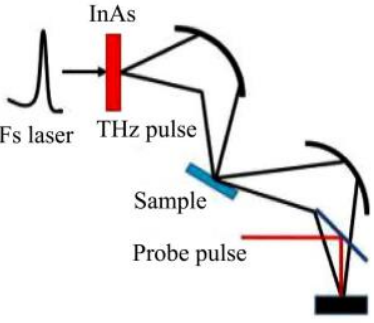

c. Reflection mode
Note: BS is the beam splitter; HWP is the half-wave plate; QWP is the quarter wave plate; $\mathrm{M}$ is the mirror; $\mathrm{PM}$ is the parabolic mirror; $\mathrm{P}$ is the polarizer; $\mathrm{A}$ is the aperture; PBS is the Wollaston prism.

Figure 1 Schematic diagram of THz-TDS system

THz-TDS is a coherent detection technology, which obtains the amplitude and phase from the terahertz pulse simultaneously ${ }^{[37]}$. The absorption coefficient $\alpha(\omega)$, refraction index $n(\omega)$, and the extinction coefficient $k(\omega)$ can be directly obtained by Fourier transform. The calculation formulas are as follows:

$$
\begin{gathered}
n(\omega)=1+\Delta \varphi(\omega) \cdot \frac{c}{\omega d} \\
\alpha(\omega)=\frac{2}{d} \ln \left\{\frac{4 n(\omega)}{T(\omega)[1+n(\omega)]^{2}}\right\} \\
k(\omega)=\ln \left\{\frac{4 n(\omega)}{T(\omega)[1+n(\omega)]^{2}}\right\} \frac{c}{\omega d}
\end{gathered}
$$

where, $T(\omega)$ is the amplitude ratio of the reference spectrum to the sample spectrum; $\varphi(\omega)$ is the phase difference between the reference spectrum and the sample spectrum; $\omega$ is the frequency; $c$ is the speed of light, and $d$ is the thickness of the sample.

\subsection{Terahertz imaging technology}

Terahertz wave can be used for imaging as other forms of electromagnetic radiations ${ }^{[38]}$. With the properties of high permeability, non-destruction and fingerprint spectrum, terahertz imaging is more advantageous than other imaging methods ${ }^{[39]}$. The first terahertz imaging system was established by adding a 
two-dimensional scanning platform to the THz-TDS system by $\mathrm{Hu}$ and Nuss in $1995^{[40]}$. With the rapid development of terahertz imaging technology, the new techniques have emerged such as two-dimensional electro-optic sampling imaging, tomography, terahertz TDS imaging, terahertz near-field imaging, and continuous wave $(\mathrm{CW})$ terahertz imaging ${ }^{[41,42]}$. terahertz source, the $\mathrm{CW}$ terahertz imaging provides higher radiation intensity, higher resolution and faster speed.

\section{Theoretical model for calculation of leaf water content}

The thermogravimetric measurement is the most prominent method to calculate the water content in plant leaves, which has high reliability as well as ease operation. The water content of the plants can be calculated according to the difference of the fresh and fully dried weight of the plants ${ }^{[16]}$. However, the method is destructive and not competent for the long-term study of the same plant. The state-of-the-art theoretical models including effective medium theory, water content index and attenuation law of Lambert-Beer, are proposed to calculate water content. The
Compared to the traditional terahertz imaging with a pulse

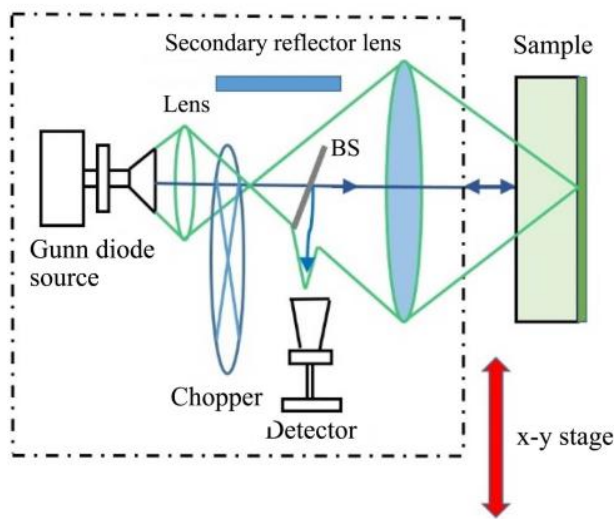

Figure 2 Schematic diagram of $\mathrm{CW}$ terahertz imaging system

Furthermore, it does not require a pump-probe system and a time-delay scan. As a result, the complexity of the optics system of $\mathrm{CW}$ can be reduced. A typical $\mathrm{CW}$ terahertz imaging system is shown in Figure 2. The terahertz beam is emitted from a Gunn diode oscillator and focused to a spot modulated by a chopper, then it is focused by the lens. The detector is made of pyroelectric detector or Schottky diode. The sample is placed on a two-dimensional translation stage controlled by a computer, and then the two-dimensional imaging of the sample can be scanned.

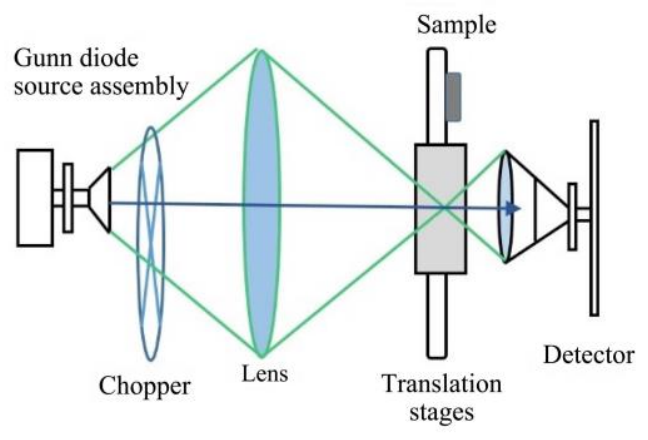

optical parameters of terahertz system and the attribute parameters of the tested leaf are used for the calculation of water content. These methods are nondestructive and of high efficiency.

\subsection{Effective medium theory}

Jördens et al. ${ }^{[43]}$ demonstrated that the dielectric properties of the leaf can be utilized to determine the water content and hence monitor drought stress in the plant. The effective medium theory model is used as a nondestructive dielectric function to extract the volumetric fraction of water in leaf tissue from THz-TDS data. As illustrated in Figure 3, a leaf is a complex and heterogeneous structure made up of water, air and dry tissue. The solid tissue is mostly composed of proteins, sugars, and other materials.
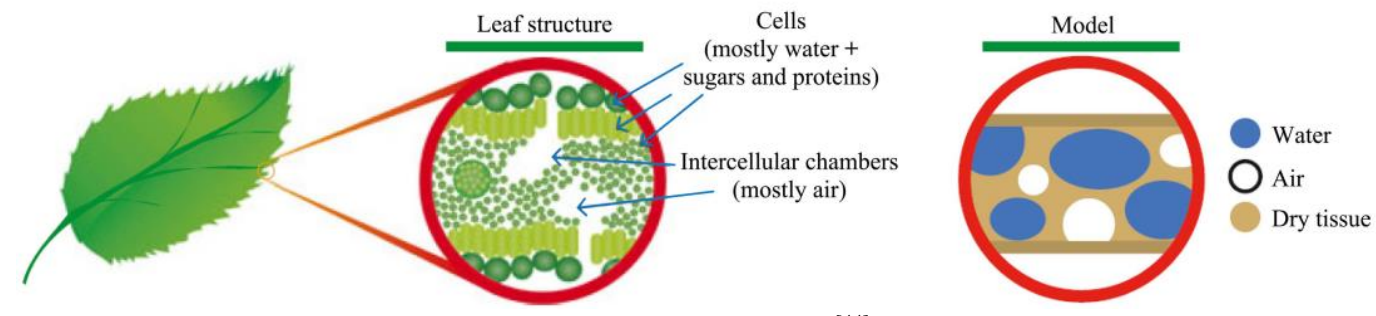

Figure 3 Leaf structure ${ }^{[44]}$
The extended Landau-Lifshitz-Looyenga model is commonly used as an effective medium approximation to obtain the terahertz dielectric function of heterogeneous mixture. And the dielectric function of the leaf is shown as follows:

$$
\sqrt[3]{\varepsilon_{L}(f)}=\xi_{W} \sqrt[3]{\varepsilon_{W}(f)}+\xi_{S} \sqrt[3]{\varepsilon_{S}(f)}+\xi_{A} \sqrt[3]{\varepsilon_{A}(f)}
$$

where, $\xi$ is the concentration of the component and $\varepsilon$ is the dielectric constant. $\quad L, W, S$ and $A$ refers to the leaves, water, solid materials and air, respectively. The permittivity of solid leaf materials can be measured by drying and compressing a leaf sample. The dielectric constant of water can be detected by a double Debye model (Equation (5)).

$$
\varepsilon_{W}(v)=\varepsilon_{\infty}+\frac{\varepsilon_{0}-\varepsilon_{1}}{1 \pm 2 \pi i v \tau_{1}}+\frac{\varepsilon_{1}-\varepsilon_{\infty}}{1 \pm 2 \pi i v \tau_{2}}
$$

where, $\varepsilon_{\infty}$ is the high frequency limit of the permittivity; $\varepsilon_{0}$ and $\varepsilon_{1}$ are constants; $\tau_{1}$ and $\tau_{2}$ are the time constants that refer to temperature-dependent slow and fast relaxation processes respectively. The \pm sign suggests that the resulting imaginary contribution to the permittivity can be either positive or negative. It depends upon the definitions. Since the definitions of the complex dielectric coefficient and complex refractive index vary in the scientific literature. The volume fraction of solid plant tissues $\left(\xi_{S}\right)$, water $\left(\xi_{W}\right)$ and air $\left(\xi_{A}\right)$ can be calculated by Equation (6):

$$
\xi_{S}=\frac{T_{3}}{T_{1}}, \xi_{W}=\frac{M_{1}-M_{2}}{T_{1} \rho_{W} A}, \xi_{A}=1-\xi_{S}-\xi_{W}
$$

where, $\rho_{W}$ is the specific gravity of water; $A$ is the leaf area; $T$ is the leaf thickness and $M$ is the leaf weight. The index $1,2,3$ indicates the measured values before drying, after drying, and after pressurization, respectively.

\subsection{Water content index}

Hunt et al. ${ }^{[45]}$ proposed a new leaf water content index (WCI) to directly correlate sample reflectance to relative water content. 
The basic principle of WCI is that the reflectance difference at a particular frequency band between a dry sample and a fresh sample is determined by the absorbance of water in that sample ${ }^{[46]}$. Two different frequencies are selected, the one is strongly absorbance of water while another one is insensitive to the change in water content. The reflectance difference obtained at different frequency bands is then normalized. Comparing this difference makes it possible to normalize the values so as to calculate the corresponding water content. WCI is defined as:

$$
W C I_{T H z}=\frac{-\ln \left[1-\left(\sin ^{2}(r)_{\Delta f T H z 1}^{i n-\text { situ }}-\sin ^{2}(r)_{\Delta f T H z 2}^{\text {in-situ }}\right)\right]}{-\ln \left[1-\left(\sin ^{2}(r)_{\Delta f T H z 1}^{\text {full-turgor }}-\sin ^{2}(r)_{\Delta f T H z 2}^{\text {full-turgor }}\right)\right]}
$$

where, The subscripts 1 and 2 refer to the two terahertz frequencies at which the reflectivity is measured. The superscript full-turgor refers to measurements with a sample at full turgor, where water content is maximum and the plant cells are full of water and resist further storage of water. According to Equation (7), the precision of WCI can be improved by choosing two terahertz frequencies with large difference in water absorption.

\subsection{Lambert-Beer attenuation law}

The attenuation law of Lambert-Beer can be applied as a theoretical method for calculating the water content from terahertz imaging ${ }^{[47]}$. The basic idea is that the leaf sample can be assumed as composed of small cubes with the procedure of terahertz scanning ${ }^{[48]}$.

$$
I(v)_{i}=I_{0}(v)_{i} \exp [k(v)] c_{i} l_{i}
$$

where, $v$ is the frequency of terahertz wave; $I_{0}(v)_{i}$ and $I(v)_{i}$ are the terahertz radiation intensities before and after penetrating the sample; $k(v)$ is the absorption coefficient of the sample; $c_{i}$ is the volume fraction of water, and $l_{i}$ is the thickness of the cube in the sample; $s_{i}$ is the length of the cube (the scanning step), the volume of the cube is $s_{i} \times s_{i} \times l_{i}$. The water content in one cube can be calculated according to Equation (9):

$$
M_{i}=\frac{s_{i}^{2}}{k(v)} \ln \frac{I_{0}(v)_{i}}{I(v)_{i}}
$$

The water content of the sample can be calculated by summing up all the cubes:

$$
M=\sum M_{i}=\sum \frac{s_{i}^{2}}{k(v)} \ln \frac{I_{0}(v)_{i}}{I(v)_{i}}
$$

As expressed in Equation (10), the attenuation law of Lambert-Beer can be efficiently applied to calculate the water content in the plant leaves by recording the parameters of terahertz imaging.

\section{Two ways of eliminating scattering effect}

The roughness and geometry of the leaf surface differ from species to species of the plant, and there is no fixed ratio between the reflection and scattering ${ }^{[49,50]}$. In order to obtain accurate and reliable results, the surface scattering of the leaves needs to be taken into account. The theoretical method based on the effective attenuation coefficient, and the practical method based on the improvement of the instrument, are used to compensate the scattering loss.

\subsection{Effective attenuation coefficient}

According to the research work of Jördens et al. ${ }^{[43,51]}$, the scattering loss has a crucial influence on the absorption, especially when the frequency is higher than $1 \mathrm{THz}$. A Rayleigh roughness factor is implemented to describe the influence of scattering due to surface roughness. To obtain more accurate measurement results, scattering caused by the roughness of layers in a host material and its effect on terahertz attenuation must be explicitly considered. Therefore, the total absorption coefficient can be expressed as the sum of the detected absorption and scattering:

$$
\begin{gathered}
\alpha_{\mathrm{res}}=\alpha_{\text {abs }}+\alpha_{\text {scat }} \\
\alpha_{\text {scat }}=\left(\Delta \varepsilon(f) \cdot \frac{4 \pi \tau \cos (\theta)}{\lambda}\right)^{2} \times \frac{1}{T} \\
\Delta \varepsilon(f)=\sqrt{\varepsilon_{L}(f)}-1
\end{gathered}
$$

where, $\theta$ is the incident angle; $\lambda$ is the wavelength, and $T$ is the leaf thickness; $\tau$ is the degree of surface roughness expressed by the standard deviation, which can be measured by determining several height profiles from different points of a leaf; $\varepsilon_{L}(f)$ is the dielectric function of the leaf as shown in Equation (4).

\subsection{Instrument improvement}

The detector of the general terahertz spectroscopy or imaging system is installed at a fixed angle. Hence, the scattered radiation of the sample from other angles cannot be detected. To solve this problem, Gente et al. ${ }^{[52]}$ and Ralf and Martin ${ }^{[53]}$ designed a new system, where the detector can be moved around a barley plant within an angular range of $270^{\circ}$ to compensate the scattering loss. As shown in Figure 4, the plant is placed in the middle of the setup, the emitter is kept in fixed positions, and the detector scans around the plant on a motorized arm. In this way, the transmitted and scattered radiation of the plant can be integrated from different angles. Gente's work has also demonstrated that the biggest part of radiation is captured in the vertical direction. However, the scattering loss from other angles occupied a significant amount, and the results of the measurements would be distorted if the scattered parts were omitted. Hence, the detection accuracy of $\mathrm{THz}$ can be improved by applying this modified terahertz system.

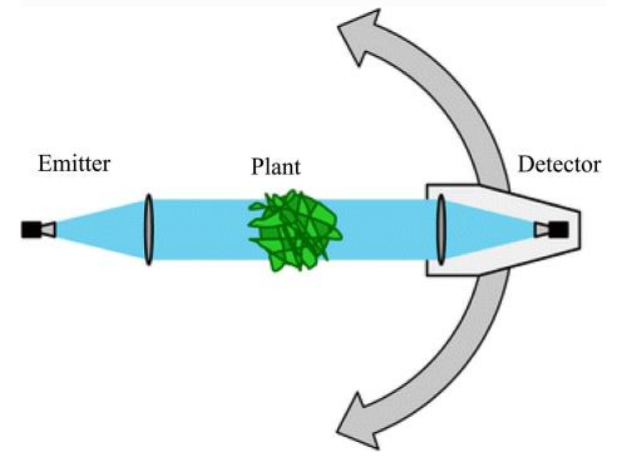

Figure 4 Schematic of the geometry of the measurement $\operatorname{setup}^{[52,53]}$

\section{Research aspects of leaf water detection}

The relatively high permittivity of liquid water compared to other materials in terahertz range promotes an explosion of researches and applications of water content detection and water distribution imaging. This section highlights some appealing research aspects, including the research of the relationship between leaf thickness and water content, monitoring of water status under the influence of the external environment, identification of plant species and planting conditions, and description of water content and water distribution. Among them, with the help of good theoretical models and ways of Eliminating scattering, some studies have made smooth progress, which provided a good basis for the following research work.

\subsection{Researching the relationship of leaf thickness and water content}

Leaf is the most important organ of plants, and its 
morphological change can reflect the change of plant growth state. The plant leaf is mainly composed of veins, petiole and mesophyll, etc. The water and other nutrients can be transported through the veins. The mesophyll is mainly composed of cells and chlorophyll, which serves as the main place for photosynthesis. The water content in veins and mesophyll can be used as the evaluation index of leaf water status. Some studies have shown that the variation of leaf thickness has a certain periodicity, which can be roughly divided into two types: long period and short period. It is of great significance to figure out these regularities for studying the plant water status. Born et al. ${ }^{[54]}$ measured the water content in the main-vein of silver fir (Abies alba) seedlings by terahertz transmission spectrum with frequency between $0.1 \mathrm{THz}$ and $1 \mathrm{THz}$. The experimental results showed that the transmission varied along the main axis of a single needle due to the variation of thickness. Jördens et al. ${ }^{[43,51]}$ used the terahertz spectrum with frequency between $0.3 \mathrm{THz}$ and $1.8 \mathrm{THz}$ to study the relationship between the leaf thickness and the water content (Figure 5). The feasibility of the third-order extended medium model based on Landau, Lifshitz and Looyenga models in this band was verified. Besides, with the model, leaf water content was well calculated and scattering effect was eliminated. The results showed that the thickness of the coffee leaf (Coffea arabica L.) increased continuously with the water content, which implied that the thickness could be used as an index to evaluate the leaf water content. The similar results had also been presented by Hadjiloucas et al. ${ }^{[55,56]}$. The applications showed that leaf thickness could be used to measure the leaf water content roughly. The results might be inaccurate if the leaf thickness was used alone to evaluate the water content. Therefore, the leaf thickness could be used in combination with other indicators to improve the measuring accuracy of water content.

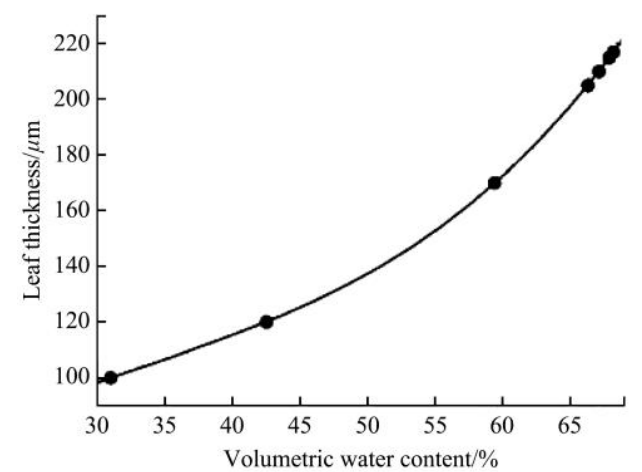

Figure 5 Leaf thickness of a coffee leaf at different volumetric water contents $^{[43]}$

6.2 Monitoring leaf water status under the influence of external conditions

External water and light conditions make important effects on the physiological conditions of plants. Too much or too little water in the soil will make the plant wilting. Plants need sunlight to carry out photosynthesis, only in organic matter, to maintain their own survival. Plants need light to photosynthesis, to create organics, and to maintain their own survival. Therefore, it is of great significance to research the leaf water content under drought stress and the effects of light on left water content. Firstly, this part summarizes the research on plant drought stress carried out by many researchers. Secondly, the studies of effect of light on leaf water content were summarized.

Monitoring plant water stress is of high importance for the basic research of plant biology and irrigation. Jördens et al. ${ }^{[43,51]}$ performed a long-term study to investigate the stressed status of the Coffea Arabica plant. The volumetric water contents were detected for a period of 21 days. The results showed that as time went by, the stress degree caused by drought increased, the transmission (at $0.3 \mathrm{THz}$ ) increased significantly, while the water content decreased significantly. Breitenstein et al. ${ }^{[34]}$ conducted a long-term measurement to monitor the changes of leaf water content during drought stress and the re-hydration after re-watering. The results showed that there was a good correspondence between the decreased water and increased transmittance in coffee plant leaves. The similar results have also been reported by Jördens et al. ${ }^{[43]}$ and Gente et al ${ }^{[57]}$. Born et al. ${ }^{[54]}$ used the changes of $\mathrm{THz}$ transmission spectrum to characterize the changes of water content. The reactions of vivo plants in a drought stress or recovery after irrigation were detected by monitoring the changes of transmission spectrum. The results implied that the changes of $\mathrm{THz}$ transmittance could be effectively used to reflect the changes of water content in plant leaves. In addition, the response of $\mathrm{THz}$ spectrum was sensitive and acute to the change of leaf water content, which could be applied to reveal the physiological state of plants.

The stomata of the plant leaves will open under light. Then the processes of photosynthesis, transpiration and respiration will increase the consumption of water. The water consumption of the plant leaves will be reduced without light, and roots will absorb water from the soil. Ralf and Martin ${ }^{[53]}$ and Gente et al. ${ }^{[57]}$ performed a long-term detection of the water status of rye (Secale cereale) plants using terahertz transmission spectrum. The differences of transmission between daytime and nighttime were monitored. Higher transmissions were detected during daytime, which was caused by the higher usage of water and lower water content left in leaves. Lower transmissions were detected during nighttime, which was caused by the higher water content in leaves due to water absorption from soil. Rehn et al. ${ }^{[58]}$ monitored the difference of drought stress responses of two plants by terahertz. The results showed that the difference between day and night of both plants was evident. The water status changed alternately from daytime to nighttime, which reflected the impacts of light on leaf water status. Castrocamus et al. $^{[59]}$ used terahertz spectroscopy (0.1-3 THz) to study the effect of dark-light cycles on the water dynamics of Arabidopsis thaliana. The results demonstrated that the changes of the average absorption coefficient are sensitive enough to reflect the variation of leaf water content caused by the opening of stomata in light and the closing of stomata in dark. Norman et al. ${ }^{[60]}$ conducted a long-term measurement of the $\mathrm{THz}$ transmission spectrum through a leaf of a rapeseed plant. The transmission values were calculated out of the mean values in a frequency band from 150-300 GHz. As shown in Figure 6, the dependency of dehydration on the day-night cycle and a full recovery after irrigation could be obviously observed. The cause-effect relationships in the field of botany could be studied by applying such measurements.

\subsection{Identifying plant species and planting conditions}

There are obvious differences in dehydration response of the leaves from different plant species. The difference can also be found from the same plants under different planting conditions. The terahertz technology can be applied for qualitative identification of plant species, origins and planting conditions. Hadjiloucas et al. ${ }^{[56]}$ performed a broad-band measurement of terahertz transmittance (0.1-0.5 THz) for two different leaves under various conditions. Five leaf samples were collected. Three of 
them belonged to the plant of Fatsia japonica. The rest two samples belonged to the plant of Phormium tenax. The leaf samples were under different water content levels and there were obvious differences between their terahertz spectra. The transmittances of Phormium tenax were lower than that of Fatsia japonica, which implied that Phormium tenax has better water storage ability than Fatsia japonica. The plant species and the different water status could be identified by monitoring the terahertz spectra of plant leaves, which could hardly be distinguished by other methods such as visual inspection. Rehn et al. ${ }^{[58]}$ performed the water status measurements by using terahertz quasi time domain spectroscopy (THz-QTDS). The drought stresses of rye (Secale cereale) and soy (Glycine max) were detected. During the long-term detection, the soy plant showed faster response to the drought stress than rye. The terahertz spectra could not only reflect the drought resistance ability of plants, but also be able to be used as an indicator to distinguish the plant species. Castro-Camus et al. ${ }^{[59]}$ compared the water retention capacity of the leaves of Arabidopsis plants growing in two substrates. The results showed that the plant growing in Turface showed faster dehydration rate compared with that growing in Metronix. Norman et al. ${ }^{[60]}$ compared the drought stress responses of the flower and the leaf of two similarly treated pansies (Viola spec.). Figure 7 showed the measured spectral responses. Both plants were re-watered at the end of day two (daytime). The leaf reacted immediately; the flower did not react until the end of daytime. The results implied the potential of terahertz to identify the same plant under different planting conditions. Additionally, the $\mathrm{THz}$ method could allow comparisons between the response of different leaf areas, different leaf ages and even the different stress responses. The comparison between different genotypes or taxa may be of interest for future cultivation of agriculture.

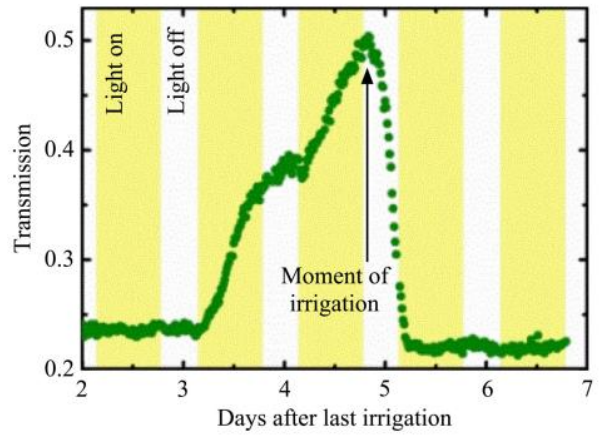

Figure 6 Monitored mean transmission values through a rapeseed leaf with a simulated day-night cycle in a drought stress experiment $^{[60]}$

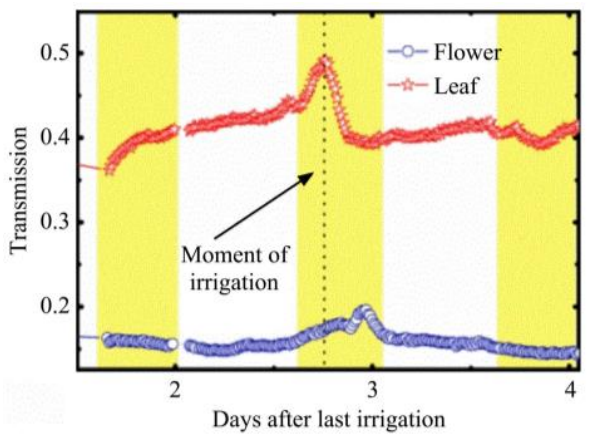

Figure 7 Monitored mean transmission values through a leaf and a flower of two different pansies with a drought stress experiment (adapted from [60])

\subsection{Terahertz imaging of leaf water content and distribution}

The first terahertz time-domain image was obtained by scanning a drying leaf ${ }^{[40]}$. The relatively high permittivity of water compared to other materials in the $\mathrm{THz}$ range enables a contrast mechanism for the detection and imaging of water ${ }^{[61]}$. In addition, there is a high contrast in the image between "moist" and "dry" regions. Therefore, the water content and its distribution in the leaves could be clearly distinguished by the Terahertz imaging. Yuichi Ogawa et al. ${ }^{[62]}$ detected the moisture change of a Hedera helix leaf using a transillumination terahertz imaging system. The change of the moisture distribution was clearly visible, which showed the ability of terahertz imaging for monitoring the water content in plants. The high lateral resolution capability of $\mathrm{THz}$ on a fresh green leaf was also illustrated by de Cumis et al. ${ }^{[63]}$. The high contrast was achievable due to the presence of water in the very minute veins. Figure 8 a displayed the photograph of the leaf sample. Figure $8 \mathrm{~b}$ depicted the $\mathrm{THz}$ image of the entire sample. The size of the covered area was $1.8 \mathrm{~mm} \times 3.2 \mathrm{~mm}$ and the resolution was $90 \times 160$ pixels, and each point with a step of $200 \mu \mathrm{m}$ long. Figure $8 \mathrm{c}$ showed more significantly details of the closed-up area inside the white rectangular frame in Figure $4 \mathrm{~b}$. The resolution was $200 \times 200$ pixels, and each point with a step of $50 \mu \mathrm{m}$ long. The water in the small veins were observed from the $\mathrm{THz}$ image. Zhang et al. ${ }^{[64]}$ proposed a quantitative method to calculate water content of the spinach leaf based on terahertz imaging at frequency of $0.189 \mathrm{THz}$. A total of 4 terahertz images were taken at room temperature in different days. The algorithm of Lambert-Beer attenuation law was used in the terahertz images to calculate the water content. The results of the calculated values of water content were quite similar to the measured values. It showed that the leaf water content and distribution could simply be measured by terahertz imaging.

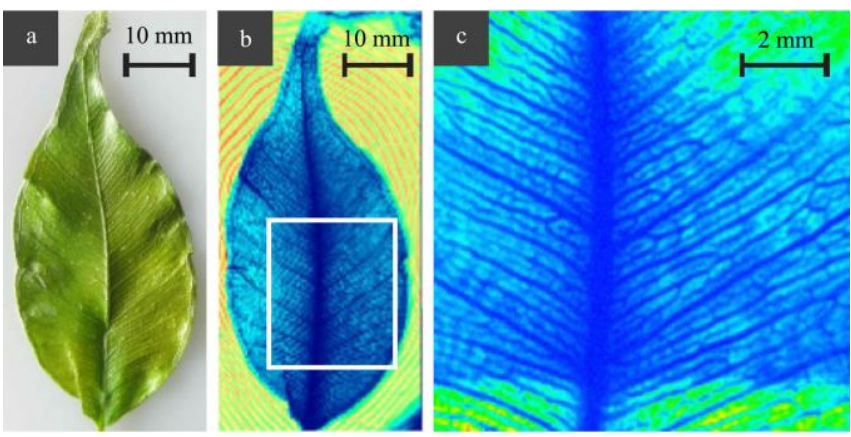

Figure 8 High resolution terahertz images of a fresh leaf $(\mathrm{a})^{[63]}$, the confocal terahertz image of the leaf (b) and the highlight $\mathrm{THz}$ image inside the closed-up area $(c)^{[63]}$

\section{Conclusions}

The property of water absorption makes $\mathrm{THz}$ an effective tool for leaf water monitoring in the field of agriculture. The basic information of terahertz radiation and the typical terahertz spectroscopy and imaging systems were summarized. The theoretical models for nondestructively calculating water content and the methods of eliminating scattering loss of leaf surface were illustrated. The studies of exploring terahertz spectroscopy and imaging in the applications of monitoring leaf water status were reviewed. The survey of the researches implied great potential of terahertz in moisture detection for plants. The response mechanism of water stress and illumination, as well as the physiological indicators of the plants, including the water retention, 
dehydration, osmotic potential, can be revealed by terahertz. In addition, terahertz technology can be extended to the identification of plant varieties, living conditions and origins. It provides a new technical solution for the development of the modern agriculture and shows broad application prospects and economic benefits.

\section{Acknowledgements}

This work was supported by the National Key Point Research and Invention Program of the Thirteenth (2016YFD0700304) and the National Key Research \& Development program of China (2016YFD0300606 and 2017YFD0700501).

\section{[References]}

[1] Signorelli S, Corpas F J, Borsani O, Barroso J B, Monza J. Water stress induces a differential and spatially distributed nitro-oxidative stress response in roots and leaves of Lotus japonicus. Plant Sci, 2013; 201-202(3): 137-146.

[2] Gomez-Bellot M J, Nortes P A, Ortuno M F, Romero C, Fernandez-Garcia N, Sanchez-Blanco M J. Influence of arbuscular mycorrhizal fungi and treated wastewater on water relations and leaf structure alterations of Viburnum tinus L. plants during both saline and recovery periods. J. Plant Physiol, 2015; 188: 96-105.

[3] Ullah S, Skidmore A K, Ramoelo A, Groen T A, Naeem M, Ali A. Retrieval of leaf water content spanning the visible to thermal infrared spectra. ISPRS Journal of Photogrammetry \& Remote Sensing, 2014; 93(7): 56-64.

[4] Cheng T, Rivard B, Sánchez-Azofeifa A G, Féret J B, Jacquemoud S, Ustin S L. Predicting leaf gravimetric water content from foliar reflectance across a range of plant species using continuous wavelet analysis. J. Plant Physiol, 2012; 169(12): 1134-1142.

[5] Püschel D, RydlováJ, Vosátka M. Can mycorrhizal inoculation stimulate the growth and flowering of peat-grown ornamental plants under standard or reduced watering? Applied Soil Ecology, 2014; 80: 93-99.

[6] Girón I F, Corell M, Galindo A, Torrecillas E, Morales D, Dell'Amico J, et al. Changes in the physiological response between leaves and fruits during a moderate water stress in table olive trees. Agricultural Water Management, 2015; 148: 280-286.

[7] ie P, Qu F, Lin L, Dong T, He Y, Shao Y. Detection of water content in rapeseed leaves using terahertz spectroscopy. Sensors, 2017; 17(12): 2830.

[8] Rodríguez P, Mellisho C D, Conejero W, Cruz Z N, Ortuño M F, Galindo A, et al. Plant water relations of leaves of pomegranate trees under different irrigation conditions. Environ. Exp. Bot, 2012; 77: 19-24.

[9] Muramatsu N, Hiraoka K. Estimation of water content in the leaves of fruit trees using infrared images. Horticultural Research, 2006; 5(5): 397-402.

[10] Federici J F. Review of moisture and liquid detection and mapping using terahertz imaging. Journal of Infrared, Millimeter, and Terahertz Waves, 2012; 33(2): 97-126.

[11] Ferrante D, Oliva G E, Fernández R J. Soil water dynamics, root systems, and plant responses in a semiarid grassland of Southern Patagonia. Journal of Arid Environments, 2014; 104: 52-58.

[12] Gerhards M, Rock G, Schlerf M, Udelhoven T. Water stress detection in potato plants using leaf temperature, emissivity, and reflectance. International Journal of Applied Earth Observation \& Geoinformation, 2016; 53: 27-39.

[13] Ni Z, Liu Z, Huo H, Li Z L, Nerry F, Wang Q, et al. Early water stress detection using leaf-level measurements of Chlorophyll fluorescence and temperature data. Remote Sensing, 2015; 7(3): 3232-3249.

[14] Cheng T, Riaño D, Koltunov A, Whiting M L, Ustin S L, Rodriguez J. Detection of diurnal variation in orchard canopy water content using MODIS/ASTER airborne simulator (MASTER) data. Remote Sens. Environ, 2013; 132(6): 1-12.

[15] Knani S, Aouaini F, Bahloul N, Khalfaoui M, Hachicha M A, Ben Lamine A, et al. Modeling of adsorption isotherms of water vapor on Tunisian olive leaves using statistical mechanical formulation. Physica A, 2014; 400: $57-70$.

[16] Cao Z, Wang Q, Zheng C. Best hyperspectral indices for tracing leaf water status as determined from leaf dehydration experiments. Ecol. Indic, 2015; 54: 96-107.

[17] Jia J, Ji H. Plant leaf water detection instrument based on near infrared spectroscopy. International Conference on Computer and Computing Technologies in Agriculture, 2011; pp.20-27.

[18] Capitani D, Brilli F, Mannina L, Proietti N, Loreto F. In situ investigation of leaf water status by portable unilateral nuclear magnetic resonance. Plant physiology, 2009; 149(4): 1638.

[19] Hosako I, Sekine N, Patrashin M, Saito S, Fukunaga K, Kasai Y, et al At the dawn of a new era in terahertz technology. Proc. IEEE, 2007; 95(8): 1611-1623

[20] Siegel P H. Terahertz technology. IEEE Transactions on Microwave Theory \& Techniques, 2002; 50(3): 910-928.

[21] Qu F, Lin L, He Y, Nie P, Cai C, Dong T, et al. Terahertz multivariate spectral analysis and molecular dynamics simulations of three pyrethroid pesticides. Journal of Infrared Millimeter \& Terahertz Waves, 2018; 3:1-14.

[22] Karpowicz N, Zhong $\mathrm{H}$, Xu J, Lin K, Hwang J S, Zhang X C. Comparison between pulsed terahertz time-domain imaging and continuous wave terahertz imaging. Semiconductor Science \& Technology, 2005; 20(7): 293-299.

[23] Rivas J G, Schotsch C, Bolivar P H, Kurz H. Enhanced transmission of $\mathrm{THz}$ radiation through subwavelength holes. Physical Review B Condensed Matter, 2003; 68(20): 201306.

[24] Galvão R, Hadjiloucas S, Bowen J, Coelho C. Optimal discrimination and classification of $\mathrm{THz}$ spectra in the wavelet domain. Opt. Express, 2003; 11(12): 1462-1473.

[25] Ferguson B, Zhang X C. Materials for terahertz science and technology. Physics, 2003; 1(1): 26-33.

[26] Walther M, Plochocka P, Fischer B, Helm H, Uhd J P. Collective vibrational modes in biological molecules investigated by terahertz time-domain spectroscopy. Biopolymers, 2002; 67(4-5): 310-313.

[27] Qu F, Pan Y, Lin L, Cai C, Dong T, He Y, et al. Experimental and theoretical study on terahertz absorption characteristics and spectral de-noising of three plant growth regulators. Journal of Infrared Millimeter \& Terahertz Waves, 2018; 1-13.

[28] Qu F, Lin L, Cai C, Dong T, He Y, Nie P. Molecular characterization and theoretical calculation of plant growth regulators based on terahertz time-domain spectroscopy. Applied Sciences, 2018; 8(3): 420.

[29] Huang L, Shabaev A, Lambrakos S G, Bernstein N, Jacobs V, Finkenstadt $\mathrm{D}$, et al. Dielectric response of high explosives at thz frequencies calculated using density functional theory. J. Mater. Eng. Perform, 2012; 21(7): 1120-1132.

[30] Hehre W J. Ab initio molecular orbital theory. Wiley, 1986; p 399-406.

[31] Zhang F, Wang H W, Tominaga K, Hayashi M. Characteristics of low-frequency molecular phonon modes studied by $\mathrm{THz}$ spectroscopy and solid-state Ab initio theory: Polymorphs I and III of diflunisal. J. Phys. Chem. B, 2016; 29(4): 41-62.

[32] Paloscia S, Santi E. A semi-empirical algorithm for estimating soil moisture from dual-frequency microwave AMSR data. Geoscience and Remote Sensing Symposium (IGARSS 03), IEEE, 2003; pp.677-679.

[33] Qu F, Lin L, He Y, Nie P, Cai C, Dong T, et al. Spectral characterization and molecular dynamics simulation of pesticides based on terahertz time-domain spectra analyses and density functional theory (DFT) calculations. Molecules, 2018; 23: 1607.

[34] Breitenstein B, Scheller M, Shakfa M K, Kinder T, Müller-Wirts T, Koch $\mathrm{M}$, et al. Introducing terahertz technology into plant biology: A novel method to monitor changes in leaf water status. Journal of Applied Botany and Food Quality, 2011; 84(2): 158-161.

[35] Kuroda R, Sei N, Oka T, Yasumoto M, Toyokawa H, Ogawa H, et al. Development of high power THz-TDS system based on S-band compact electron linac. Radiation Physics \& Chemistry, 2008; 77(10): 1131-1135.

[36] Mendis R, Sydlo C, Sigmund J, Feiginov M, Meissner P, Hartnagel H L. Tunable CW-THz system with a log-periodic photoconductive emitter. Solid-State Electron, 2004; 48(10-11): 2041-2045.

[37] Duvillaret L, Garet F, Coutaz J L. A reliable method for extraction of material parameters in terahertz time-domain spectroscopy. IEEE J. Sel. Top. Quantum Electron, 1996; 2(3): 739-746.

[38] Usami M, Iwamoto T, Fukasawa R, Tani M, Watanabe M, Sakai K. Development of a THz spectroscopic imaging system. Phys. Med. Biol, 2002; 47(21): 3749-53.

[39] Chen H T, Kersting R, Cho G C. Terahertz imaging with nanometer resolution. Appl. Phys. Lett, 2003; 83(15): 3009-3011.

[40] Hu B B, Nuss M C. Imaging with terahertz waves. Opt. Lett, 1995; 20(16): 1716 
[41] Siebert K J, Quast H, Leonhardt R, Löffler T, Thomson M, Bauer T, et al. Continuous-wave all-optoelectronic terahertz imaging. Appl. Phys. Lett, 2002; 80(16): 3003-3005.

[42] Dobroiu A, Yamashita M, Ohshima Y N, Morita Y, Otani C, Kawase K. Terahertz imaging system based on a backward-wave oscillator. Appl. Opt, 2004; 43(30): 5637-5646.

[43] Jördens C, Scheller M, Breitenstein B, Selmar D. Evaluation of leaf water status by means of permittivity at terahertz frequencies. J. Biol. Phys, 2009; 35(3): 255-264.

[44] Gente R, Born N, Voß N, Sannemann W, Léon J, Koch M, et al. Determination of leaf water content from terahertz time-domain spectroscopic data. Journal of infrared, Millimeter, and Terahertz Waves, 2013; 34(3): 316-323.

[45] Erjr H, Rock B N, Nobel P S. Measurement of leaf relative water content by infrared reflectance. Remote Sens. Environ, 1987; 22(3): 429-435.

[46] Hadjiloucas S, Walker G C, Bowen J W, Zafiropoulos A. Propagation of errors from a null balance terahertz reflectometer to a sample's relative water content. Journal of Physics Conference Series, 2009; p.12.

[47] Stavn R H. Light attenuation in natural waters: Gershun's law, Lambert-Beer law, and the mean light path. Appl. Opt, 1981; 20(14): 2326-2327.

[48] Zhang H B, Mitobe K, Yoshimura N. Application of Terahertz imaging to water content measurement. Japanese Journal of Applied Physics, 2008; 47(10): 8065-8070

[49] Scheller M, Jordens C, Breitenstein B, Selmar D, Koch M. Effective permittivity and scattering model for the evaluation of the leaf water status, 33rd International Conference on Infrared, Millimeter and Terahertz Waves (IRMMW-THz 2008), 2008; pp.1-2.

[50] And D T, Söderman O. Diffusion of water absorbed in cellulose fibers studied with 1H-NMR. Langmuir, 2001; 17(9): 2694-2702.

[51] Jördens C, Wietzke S, Scheller M, Koch M. Investigation of the water absorption in polyamide and wood plastic composite by terahertz time-domain spectroscopy. Polym. Test, 2010; 29(2): 209-215.

[52] Gente R, Rehn A, Koch M. Contactless water status measurements on plants at $35 \mathrm{GHz}$. Journal of Infrared, Millimeter, and Terahertz Waves, 2015; 36(3): 1-6.

[53] Ralf G, Martin K. Monitoring leaf water content with $\mathrm{THz}$ and sub-THz waves. Plant Methods, 2015; 11:15.

[54] Born N, Behringer D, Liepelt S, Beyer S. Schwerdtfeger M, Ziegenhagen $\mathrm{B}$, et al. Monitoring plant drought stress response using terahertz time-domain spectroscopy. Plant Physiology, 2014; 164(4): 1571-1577.

[55] Hadjiloucas S, Galvão R K, Bowen J W. Analysis of spectroscopic measurements of leaf water content at terahertz frequencies using linear transforms. Journal of the Optical Society of America A Optics Image Science \& Vision, 2002; 19(12): 2495-2509.

[56] Hadjiloucas S, Karatzas L S, Bowen J W. Measurements of leaf water content using terahertz radiation. IEEE Transactions on Microwave Theory \& Techniques, 1999; 47(2): 142-149.

[57] Gente R, Born N, Velauthapillai A, Balzer J C. Monitoring the water content of plant leaves with $\mathrm{THz}$ time domain spectroscopy. International Conference on Infrared, Millimeter, and Terahertz Waves, 2015; pp 1-2.

[58] Rehn A, Gente R, Probst T, Balzer J C. Plant water status monitoring with THz QTDS. German Microwave Conference, 2016; pp.4-6.

[59] Castrocamus E, Palomar M, Covarrubias A A. Leaf water dynamics of Arabidopsis thaliana monitored in-vivo using terahertz time-domain spectroscopy. Scientific reports, 2013; 3(10): 2910-2910.

[60] Born N, Gente R, Behringer D, Schwerdtfeger M. Monitoring the water status of plants using $\mathrm{THz}$ radiation. International Conference on Infrared, Millimeter, and Terahertz Waves, 2014; 1-2.

[61] Thamboon P, Buaphad P, Thongbai C, Saisud J, Kusoljariyakul K, Rhodes $\mathrm{M} \mathrm{W}$, et al. Investigation of water distribution in proton exchange membrane fuel cells via Terahertz imaging. Nucl. Instrum. Methods Phys. Res., Sect. A, 2011; 637(1): S161-S164.

[62] Ogawa Y, Kawase K, Mizuno M, Yamashita M, Otani C. Nondestructive and real-time measurement of moisture in plant. IEEJ Transactions on Electronics Information \& Systems, 2004; 124(9): 1672-1677.

[63] de Cumis U S, Xu J H, Masini L, Degl'Innocenti R, Pingue P, Beltram F, et al. Terahertz confocal microscopy with a quantum cascade laser source. Opt. Express, 2012; 20(20): 21924-21931.

[64] Zhang H, Mitobe K, Yoshimura N. Terahertz imaging for water content measurement. International Symposium on Electrical Insulating Materials, 2008; pp.87-90. 